\title{
Quantitative Methods for a New Configuration of Territorial Units in a Chilean Government Agency Tender Process
}

\author{
Guillermo Durán \\ Departamento de Ingeniería Industrial, FCFM, Universidad de Chile, 8370439 Santiago, Chile; \\ Departamento de Matemática, FCEN, Universidad de Buenos Aires, Buenos Aires, \\ Argentina; CONICET, Argentina, gduran@dii.uchile.cl \\ Rafael Epstein, Cristian Martinez, Gonzalo Andres Zamorano \\ Departamento de Ingeniería Industrial, FCFM, Universidad de Chile, 8370439 Santiago, Chile \\ \{repstein@dii.uchile.cl, cmartinezahumada@yahoo.com, gonzaloz@dii.uchile.cl\}
}

\begin{abstract}
The Junta Nacional de Auxilio Escolar y Becas (JUNAEB) is an agency of the Chilean government with responsibility for promoting the integration and retention of socially vulnerable children in the country's school system. Its services include a school meals program under which private firms bid on supply contracts for territorial units within Chile. Before 2007, these units were defined manually and their attractiveness to potential suppliers varied. This led to a series of problems for the government, both in the contract tender process and the service provided. In this paper, we apply operations research methodologies to determine new configurations of the territorial units to ensure that their attractiveness is similar and that schools in each region of the country receive meal service of similar, good quality. This homogenization of the units' attractiveness helps reduce the uncertainty and bankruptcy risk that suppliers face, thus benefitting the entire system. Since 2007, JUNAEB has used the configurations we proposed.
\end{abstract}

Key words: combinatorial auctions; operations research; quantitative methods; territorial units. History: This paper was refereed. Published online in Articles in Advance June 1, 2011.

$\mathrm{T}^{\mathrm{s}}$ he Junta Nacional de Auxilio Escolar y Becas (JUNAEB), an agency of the Chilean government, is responsible for promoting the integration and retention of socially vulnerable children in the country's school system. The agency runs a series of programs to ensure equality of opportunity in education. Its activities reach almost two million children in public schools throughout the nation.

JUNAEB's school meals program currently operates in approximately 10,000 schools; its yearly cost to the government is USD 600 million. In an annual tender organized by the agency, food industry firms bid on supply contracts to provide the meals. The supply contracts are auctioned based on a series of disjoint, compact geographical areas called territorial units (TUs), which cover the country. A company that is awarded the contract for a TU must provide meal services to all schools in that TU. This tender is the largest competitive procurement process in Chile in either the public or private sector.
Each agency-defined TU consists of groups of "comunas" or districts, the smallest administrative division constituting Chile's 13 official regions, which extend from the 1st region (Region I) in the north to the 12th region (Region XII) in the south, plus the Metropolitan (Santiago) region. Of Chile's 346 districts, 104 (30 percent) have fewer than 10,000 inhabitants (44 have under 5,000), 195 (57 percent) are populated by 10,000 to 100,000 inhabitants, and 46 (13 percent) are home to more than 100,000.

JUNAEB grouped these districts into 136 TUs delimited based on geographical criteria plus an attempted equalization of the number of meals to be served in each TU within a given region (see Figure 1). This simplistic procedure led to major problems in the tender process for both JUNAEB and the meal suppliers because of significant disparities in the attractiveness of the TUs. Districts in regions requiring large numbers of meals were assigned to a single $\mathrm{TU}$, whereas the remaining districts were combined 


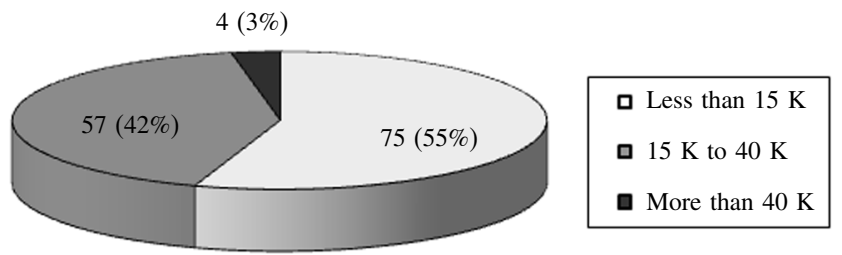

Figure 1: The pie chart shows the distribution of TUs by number of meals before 2007 .

into TUs requiring a similar quantity independent of the total area they covered or the number of schools they contained.

In our study, a TU's attractiveness is derived in terms of a score based on four characteristics (i.e., criteria) of its constituent districts-number of meals, number of schools, area, and accessibility. The number of meals to be served gives a precise measure of the magnitude of the contract; the number of schools determines the fixed cost of supplying each establishment; the area covered (in $\mathrm{km}^{2}$ ) indicates transport costs; and relatively inaccessible schools (because of geography or climate) increase supplier costs.

The objective of this paper is to describe how we applied a series of operations research (OR) methodologies to determine new configurations of TUs in each region and ensure that each unit's attractiveness level is similar.

For strategic purposes, JUNAEB established that the minimum and maximum numbers of meals for each TU in the new configuration are 15,000 and 40,000 , respectively. The lower bound considers certain agency operating considerations and the minimum quantity required to exploit scale economies; the upper bound tends to ensure that most regions will have multiple suppliers.

We use April 2007 data to quantify each of the four criteria. Although these data may fluctuate monthly, the results they generate should not differ significantly; in the short term, the relative magnitudes for the districts in a given region vary only slightly, if at all. For example, if the number of meals increases by 5 percent at the national level, the rise for each district will also be close to 5 percent.

A region in which more meals per day are served tends to have more TUs (see Table 1).

\begin{tabular}{lrr} 
Region & No. of TUs & Total meals/day \\
\hline & & \\
I & 2 & 50,350 \\
II & 2 & 39,844 \\
III & 2 & 35,114 \\
IV & 5 & 87,777 \\
V & 10 & 186,941 \\
VI & 9 & 104,187 \\
VII & 10 & 157,525 \\
VIII & 17 & 286,860 \\
IX & 9 & 158,915 \\
X & 11 & 197,087 \\
XI & 1 & 10,541 \\
XII & 1 & 12,177 \\
MR & 57 & 655,429 \\
Total & 136 & $1,982,747$
\end{tabular}

Table 1: The table shows the number of meals and TUs per region before 2007.

For each district, a series of data describes the characteristics of its schools, the number of meals served, the type of meals, the TU to which it belongs, etc. Generally speaking, the district will be the basic unit in homogenizing the TUs, given that this information completely describes it. If, however, the number of daily meals served in a district is greater than 10,000, we divide the district into two or more subdistricts; the precise number is the lowest that ensures each serves an equal quantity of meals, but not more than 10,000 . We refer to the basic unit in the formulation of our problem as a cell.

Because we defined the problem in terms of four criteria that may conflict, we require a multipleobjective decision technique. We chose a variation on the analytic hierarchy process (AHP) (Saaty 1980) to determine the weight to be given to each criterion (see the Relative Importance of a TU section).

To solve our problem, we began by developing a local search heuristic that starts with an initial TU configuration and proceeds to exchange districts between TUs, trying to minimize the standard deviation of the TU scores. To validate these outcomes a posteriori, we developed two additional methodologies: one was based on an integer linear programming (ILP) model; the second combined this model with the heuristic.

The ILP approach uses an algorithm that constructs clusters of districts to configure each TU (Goycoolea et al. 2009) subject to constraints. For each cluster, 
we calculate a score and enter it into an ILP model that attempts to minimize the difference between the highest and lowest scores within each region. In most cases, this objective yields results concordant with those generated by the heuristic's objective of minimizing standard deviation. The combined methodology also minimizes the standard deviation using the heuristic but takes the final result generated by the ILP model as its initial solution.

Other applications also use the generation of units or blocks to resolve problems; these include forest harvest scheduling, in which a maximum contiguous harvest area must be observed (Goycoolea et al. 2005, 2009; Murray and Weintraub 2002), and assigning students to schools based on residence district within a city (Caro et al. 2004, Taylor et al. 1999).

The final solutions delivered by these three methodologies for the 10 (of 13) Chilean regions covered by our study differ little from each other and substantially improve the pre-2007 situation. In 2007, JUNAEB adopted the configuration generated by the local search heuristic, which was the first part of this research completed, with slight modifications suggested by the agency for its three-year tender process (one-third of the country each year). The subsequent development of the two other methodologies confirmed that the solution JUNAEB used provides substantial benefits.

We did not include Chile's 11th and 12th regions, each of which is covered by a single TU, in this study. The number of daily meals served in each suggests that neither should be partitioned into more than a single entity. We also excluded the Metropolitan (Santiago) region, where the meals program is handled mainly by small and medium-sized businesses. JUNAEB was satisfied with this region's existing partition and opted to leave its TUs unchanged. These three regions cover 59 of the 136 original countrywide TUs.

\section{The Need for More Equally Attractive Territorial Units}

In 1997, JUNAEB officials and researchers at the University of Chile designed and implemented a combinatorial auction that allowed companies to bid on any set of TUs. The aim was to benefit from potential scale or density economies or other efficiencies, which would be reflected in the bidders' competitive prices for different TU combinations. Auction participants could submit thousands of bids, each representing a different combination of units.

To identify the best solution for the tender, the academics at the University of Chile developed a mathematical programming model that delivers the combination of bids to optimize the objective function. It incorporates the government's criteria regarding costs and quality (Epstein et al. 2002), subject to various economic, technical, and operational constraints. Each year, a third of the TUs are contracted for a three-year period, ensuring that suppliers can recoup their investments in a reasonable period.

The strategy behind the tender design is based on three pillars. First, a process of the scope of this meals program must guarantee potential bidders that it will be fair and transparent. Therefore, the optimization model was included in the tender specifications and was known to all participating suppliers. Furthermore, the bids were made public as soon as bidding was closed, permitting any company to replicate the award process to ensure complete transparency. This practice increased the competitiveness of the tender; the number of suppliers now participating is 60 percent higher than in 1997.

The second pillar promotes greater supplier operations efficiency. This was achieved by devising a combinatorial mechanism that groups TUs to incorporate scale economies and other advantages a particular firm may have for a given TU combination.

The third pillar reduces or eliminates risks that suppliers cannot control or mitigate. For example, bids must state a price per serving, which is calculated based on average student attendance. If the effective average attendance is less than that number, the supplier will incur losses because of the fixed operating costs. To mitigate this risk, firms must bid different prices for different levels of average attendance, recognizing that as this factor falls, the price per serving rises. This is significant because classes in Chilean schools are sometimes suspended because of adverse weather conditions, political demonstrations, or natural disasters (e.g., the February 2010 earthquake). The cost of assuming these risks would be higher for suppliers than for JUNAEB, thereby increasing the bid prices. 


\begin{tabular}{lcccr} 
Year & TUs affected by bankruptcies & $\begin{array}{l}\text { Original annual cost } \\
\text { (millions of dollars) }\end{array}$ & $\begin{array}{c}\text { Replacement annual cost } \\
\text { (millions of dollars) }\end{array}$ & Difference (\%) \\
\hline & & & 10.56 & 1.40 \\
2004 & $101,102,1,004,1,007,1,005,1,010$ & 2.58 & 2.92 & 13 \\
2004 & 301,302 & 9.86 & 10.82 & 10
\end{tabular}

Table 2: The data shows the increased costs associated with the replacement contracts.

Another risk-minimization strategy links the bid price to general price and food price indexes, thus stabilizing supplier operations during the contract period and mitigating large cost increases (e.g., in 2007 and 2008, world food prices jumped significantly).

Before 1997, the tender process was highly subjective and open to various pressures because of the large amounts of money involved. The discontent of the government and the participating companies eventually led to a crisis of confidence that seriously damaged the agency. The method we present was developed and adopted in response to this situation.

The system based on this method (Epstein et al. 2002) generated cost-reduction and quality-improvement benefits of 22 percent of the tender's total value. In 2002, it was awarded the IFORS Prize for OR in Development as the best application in a developing country in terms of its social impact (Epstein et al. 2004). To date, it has been used in the tendering of more than USD 3 billion of services, and is one of the most successful real-world applications of a combinatorial auction (Cramton et al. 2006, Milgrom 2007). IBM-Cplex has also used it in a case study (IBM 2009, Catalan et al. 2009).

Beginning in 2004, the Chilean food industry began to exhibit disturbing bankruptcy levels, eventually causing serious losses and other problems for the government. First, restoring meal service in TUs in which suppliers had gone bankrupt was much costlier than the original contracts, because auctioning a reduced number of units is a less competitive process; the bidders tended to be the suppliers operating in neighboring areas (Olivares et al. 2010). Moreover, the contracts are for shorter terms; therefore, the prices increase because the suppliers have less opportunity to recoup their investments. Second, any interruptions in meal service negatively affect students. Many are from low-income families, and the JUNAEB meals are a significant part of their diet.
As a result of these bankruptcies, the entire school meal policy was called into question. Table 2 shows data on three cases in which services were interrupted in 2004 and 2005.

In addition, each bankruptcy eliminates a supplier from the industry, significantly reducing competitiveness and increasing costs over the medium term (Olivares et al. 2010). Five firms declared bankruptcy between 2004 and 2007.

An investigation into the reasons behind the bankruptcies found two central causes. First, a "price war" between the companies was driven by a tender system that strongly promoted competition. Because few suppliers survived these wars, the medium-term result was less competition and higher prices. To eliminate this predatory practice, a procedure was adopted to discard tender offers that are well below the average bid. Decarolis (2009) discusses the theoretical basis of this concept. However, because of their combinatorial nature, the bids generally differed, thus complicating the task of comparing and identifying inordinately low bids. Second, some suppliers did not know that operating conditions in some TUs were significantly worse than the national average. This phenomenon is the primary motivation for our study, which attempts to demonstrate the importance of homogenizing the characteristics of the different TUs to minimize the bankruptcy risk.

The central problem is that some firms did not accurately evaluate these "bad" TUs; they submitted unrealistically low bids, leaving them unable to fulfill their contracts. Suppliers who were successful in a given sector of the country often made offers on TUs in regions they knew little about; some TUs turned out to be "bad" because of unforeseen factors (e.g., local labor costs, obtaining fresh produce, school densities, school size, and school accessibility). This asymmetry is known in the literature as the "winner's curse" (Thaler 1988). In addition, school meal 
Durán et al.: Quantitative Methods for a New Configuration of Territorial Units Interfaces $41(3)$, pp. 263-277, (c) 2011 INFORMS

Category

TUs

Below average $101,1,004,1,007,1,005,1,010,302,609,803,805,1,101$

Average

Above average

$102,301,605$

$606,607,1,321$

Table 3: The attractiveness of TUs in bids leading to bankruptcy is based on definitions given above.

contracts involve high volumes and low margins ( 2 to 3 percent of sales); thus, relatively minor problems can trigger bankruptcy.

Yet another aspect is the "limited liability" effect. This phenomenon has received much attention in risk theory, especially since the start of the financial crisis in late 2008. Applied to this case, this analysis suggests that even when suppliers are fully conscious of the high risks involved, they may submit aggressive bids to win a contract. Such firms assign one probability to a healthy and profitable operation and another to an unsustainable situation that could end up in bankruptcy. In the second scenario, an entrepreneur assumes only the costs stipulated in the contract, leaving all other costs to be borne by the system-thus, the notion of "limited liability" (Parlane 2003). The supplier calculates the expected value of the contract and, in a situation of uncertainty, takes on risks that are greater than are desirable for JUNAEB. Clearly, this market imperfection imposes significant costs on the entire school meal program, driving fundamentally viable companies into bankruptcy.

Two appendices (Appendix A: TU Criterion Weights in Local Search Heuristic and Appendix B: Cluster Weights on Each Criterion in the ILP Model) are included in an electronic companion to this paper, which is available as part of the online version at http://interfaces.pubs.informs.org/ecompanion.html.

Table 3 categorizes the attractiveness levels of the TUs involved in the bankruptcies described in Table 2. The "average" category groups units whose attractiveness index deviates no more than 3 percent from the mean. The bids that led to bankruptcy evidence a strong tendency to include relatively bad TUs.

Auction theory stresses the possibility of the winning bidder's bankruptcy or nonperformance, especially when the object of the tender is a service. Milgrom (2007) analyzes a well-known case involving a radio spectrum auction in the United States.
The effects we describe above underscore the aptness of JUNAEB's strategy to reduce the risk and uncertainty levels that potential bidders face. This shows the desirability of a method for homogenizing the operational characteristics of TUs as a way of lowering the risks and uncertainties that result in high costs for the entire system.

A configuration that generates similar TUs would also avoid distortions in the values of proposals submitted by firms bidding on combinations of attractive and unattractive units. In the past, bidders sometimes won the contract for a very attractive TU with a poor offer merely because that TU was combined with an unattractive unit that received few bids (as we noted above, the tender process awards TUs in complete sets or not at all). Because this process is a combinatorial auction, an appropriate definition of these units is essential. Olivares et al. (2010) provide an in-depth treatment of strategies for drawing up bids that bundle attractive and unattractive TUs.

\section{Relative Importance of a TU}

In this section, we describe the procedure for finding an indicator that quantifies the attractiveness to bidding meal suppliers of all possible TUs constructed by the algorithm for a given region. The degree of attractiveness of any $\mathrm{TU}$ is given by the following formula:

$U_{j, r}=a_{\text {Rac }} x_{R a c, j, r}+b_{C o l} x_{C o l, j, r}+c_{S u p} x_{S u p, j, r}+d_{A c c} x_{A c c, j, r}$.

In this formula,

$U_{j, r}=$ total score for $\mathrm{TU} j$ in region $r$

$a_{R a c}=$ importance of number of meals within the set of criteria;

$x_{R a c, j, r}=$ weight of $\mathrm{TU} j$ in region $r$ on number-ofmeals criterion;

$b_{\text {Col }}=$ importance of number of schools within the set of criteria;

$x_{\mathrm{Col}, j, r}=$ weight of TU $j$ in region $r$ on number-ofschools criterion;

$c_{S u p}=$ importance of size of TU area within the set of criteria;

$x_{S u p, j, r}=$ weight of TU $j$ in region $r$ on area criterion;

$d_{A c c}=$ importance of type of access to school within the set of criteria; 


\begin{tabular}{ll} 
Level of preference & Value \\
\hline & \\
Equally preferred & 1 \\
Equally to moderately preferred & 2 \\
Moderately preferred & 3 \\
Moderately to strongly preferred & 4 \\
Strongly preferred & 5 \\
Strongly to very strongly preferred & 6 \\
Very strongly preferred & 7 \\
Very strongly to extremely preferred & 8 \\
Extremely preferred & 9
\end{tabular}

Table 4: The data show the scale of preference for pairwise comparisons, which Saaty (1980) proposed.

$\begin{aligned} x_{A c c, j, r}= & \text { weight of } \mathrm{TU} j \text { in region } r \text { on accessibility } \\ \text { criterion. } & \end{aligned}$

As we explained in general terms above, we obtained the values for $a_{\text {Rac }}, b_{\text {Col }}, c_{\text {Sup }}$, and $d_{A c c}$ used with all of the methodologies by applying a variation on the AHP (Saaty 1980). The steps in the process are given below:

1. Develop pairwise comparisons of the decision criteria (see Table 4).

2. Create a pairwise criteria comparison matrix using the following procedure:

a. Sum the values in each matrix column.

b. Normalize the matrix by dividing each element by the sum of the numbers in its column.

c. Calculate the average of the values in each row of the normalized matrix. The result is the criteria preference vector.

Once the scores on each criterion for each TU in each region $\left(x_{R a c, j, r}, x_{C o l, j, r}, x_{S u p, j, r}\right.$, and $\left.x_{A c c, j, r}\right)$ have been obtained, the total score for each TU can then be calculated (see Appendices A and B).

Pairwise criteria comparisons (see Table 5) were established in consultation with JUNAEB personnel and supplier officials. As an example, the value of 3 in the element $(1,3)$ of the matrix (row 1 , column 3 ) indicates that the number-of-meals criterion is "moderately preferred" to the area criterion (see Table 4). When the comparisons have been made, the values for each criterion are summed (row Sum). To obtain the final percentage weight factor by criterion, we average the relative importance of the various comparisons, as

\begin{tabular}{lccccc} 
Criterion & Meals & Schools & Area & Access & Weight (\%) \\
\hline Meals & 1.00 & 1.00 & 3.00 & 3.00 & 38.07 \\
Schools & 1.00 & 1.00 & 2.00 & 3.00 & 34.22 \\
Area & 0.33 & 0.50 & 1.00 & 2.00 & 16.94 \\
Access & 0.33 & 0.33 & 0.50 & 1.00 & 10.77 \\
Sum & 2.67 & 2.83 & 6.50 & 9.00 & 100.00
\end{tabular}

Table 5: The data show comparisons of criteria and their final weight factors.

the following example shows, for the calculation of the number-of-meals criterion weight (see Table 5):

38.07

$=\left(\frac{1.00 / 2.67+1.00 / 2.83+3.00 / 6.50+3.00 / 9.00}{4}\right) * 100$.

In Table 5, the Weight column shows the final values for each criterion. The weighted importance of the number of meals is 38.07 percent, higher than for any other criterion; thus, it is the most important criterion, because it already was in the view of JUNAEB and the suppliers. These weights were validated by contrasting the attractiveness values of the TUs derived on these criteria for the years prior to 2007 with the agency's analysis of TU preference levels. That is, the values for these criteria based on the pre-2007 scenario are consistent with the assumptions of JUNAEB and the suppliers regarding TU attractiveness.

A criterion is said to be directly proportional if the greater its proportion, the more attractive the TU is. By contrast, with an inversely proportional criterion, the greater the proportion, the less attractive the TU is. The number of meals and the school accessibility (i.e., an index obtained by dividing the number of easyaccess schools by the number of difficult-to-access schools) are directly proportional criteria; the area and the number of schools are inversely proportional.

The criteria weights, $x_{\mathrm{Rac}, j, r}, x_{\mathrm{Col}, j, r}, x_{\mathrm{Sup}, j, r}$, and $x_{A c c, j, r}$ for each TU in each region, analyzed in detail in the appendices, are calculated in different ways depending on which methodology (heuristic or ILP) is used; however, they maintain similar proportions under the two approaches. To obtain the weights for the criteria, data (e.g., number of schools, school characteristics, number of meals, and area) are needed for each district. 


\section{Methodology 1: Local Search Heuristic}

The objective of the local search heuristic is to find a set of homogeneously attractive TUs within each region. A TU's attractiveness is calculated using the weights defined in Appendix A and the final values shown in Table 5. The degree of homogeneity is the standard deviation, which measures the dispersion of TU attractiveness levels by quantifying the divergence of each $\mathrm{TU}$ in a region from the regional average. The heuristic attempts to minimize this measure.

The heuristic starts with different initial solutions; in each case, it seeks a local minimum by exchanging districts between TUs within a given region. For this procedure, we define a concept of neighboring solutions and select the best local optimum as the final solution of each region.

Districts that serve more than 10,000 meals in any region are partitioned (the heuristic then works with these subdistricts as if they are complete districts) to achieve a more even distribution of meals and a set of TUs with more homogeneous attractiveness levels. In such cases, the district is partitioned into the smallest number of equal subdistricts, such that each serves an equal quantity of meals totaling no more than 10,000. A 24,000-meal district, for example, would be partitioned into three subdistricts of 8,000 meals each. Area and number of schools must also be distributed equally among these subdistricts.

For each region, the first factors to be identified are the minimum and maximum number of TUs it can contain. The calculation is made as a function of the total number of meals and must satisfy the upper and lower bounds for meals per TU, which were predetermined as 15,000 and 40,000 , respectively. Thus, if a region serves 100,000 meals, it will have at least three, and not more than six, TUs.

Once this range has been established, an initial feasible (in terms of contiguity and number of meals) solution is constructed for each possible value of the number of TUs in the region. The local search heuristic is then launched with each initial solution. The construction of the initial solutions is performed independently of the heuristic using the programmer's expert knowledge and in consultation with JUNAEB officials. In cases in which the value for the number of TUs equals the number before 2007, the configuration used as the initial solution will be that of the former configuration to the extent it is feasible relative to contiguity and number of meals. For values that differ from the one under the pre-2007 configuration, or values that are the same but where the configuration is not feasible, an initial solution must be constructed.

As the heuristic proceeds from each initial solution, it searches for local minima, advancing toward the "best" (in terms of standard deviation) neighboring solution. A solution is said to neighbor another solution if the latter is obtained from the former by transferring a district from one TU to another, while preserving both units' contiguity and maintaining the number of meals in each between 15,000 and 40,000. A local mimimum is reached when no neighboring solution will lower the current standard deviation; the definitive solution is the one with the lowest standard deviation of all the local minima.

Figures 3 and 4 illustrate the heuristic's operation for Chile's 1st region, showing the standard deviation and attractiveness of the TUs for each iteration. The final solution maintains the number of TUs in the pre2007 configuration. Because the region serves somewhat more than 50,000 meals, the solution must have at least two and no more than three TUs (see Table 6).

For the two-TU configuration, the initial solution used is the one JUNAEB used before its adoption of this heuristic in 2007, given that it satisfies the contiguity and number-of-meals conditions. Its standard deviation is 5.93. As Figure 2 shows, TU 1 covers the districts of Arica (which must be partitioned into three subdistricts), Camarones, Putre, and General Lagos; TU 2 contains the rest of the 1st region districts.

In the first iteration, the Huara district is transferred from TU 2 to TU 1 and the standard deviation drops 77 percent to 1.37 . The second iteration switches the Camiña district from TU 2 to TU 1 and finds a local minimum for the resulting configuration with a standard deviation of 0.33 , or 94 percent below that calculated for the initial solution. At this point, the heuristic stops because no additional district transfers will lower the value.

For the three-TU configuration, a new feasible initial solution with a standard deviation of 3.37 is created (see Figure 3). The first iteration reduces this figure by 50 percent by transferring the Colchane district from TU 2 to TU 1. In the second (and last) iteration, the Pica 


\begin{tabular}{|c|c|c|c|c|c|c|}
\hline Region & District & Meals & Schools & Area $\left(\mathrm{km}^{2}\right)$ & Easy access & Difficult access \\
\hline \multirow[t]{12}{*}{ 1st Region } & Arica & 25,726 & 62 & 4,799 & 62 & 0 \\
\hline & Camarones & 54 & 8 & 3,927 & 7 & 1 \\
\hline & Putre & 248 & 6 & 5,903 & 6 & 0 \\
\hline & General Lagos & 180 & 9 & 2,244 & 9 & 0 \\
\hline & Iquique & 9,155 & 38 & 2,262 & 38 & 0 \\
\hline & Alto Hospicio & 11,387 & 25 & 573 & 25 & 0 \\
\hline & Huara & 460 & 12 & 10,475 & 12 & 0 \\
\hline & Camiña & 404 & 9 & 2,200 & 9 & 0 \\
\hline & Colchane & 252 & 5 & 4,016 & 5 & 0 \\
\hline & Pica & 799 & 5 & 8,934 & 5 & 0 \\
\hline & Pozo Almonte & 1,685 & 10 & 13,766 & 10 & 0 \\
\hline & Total & 50,350 & 189 & 59,099 & 188 & 1 \\
\hline
\end{tabular}

Table 6: The table summarizes the district-level data used to calculate the TU scores for the 1st region.

district moves from TU 3 to TU 1, and the heuristic finds a local minimum with a standard deviation of 0.88 , a 74 percent reduction from the initial situation.

Finally, the standard deviations of the two local minima are compared; the lowest value indicates the definitive TU configuration for the region. In this example, the final solution is the two-TU configuration.

We programmed the heuristic in Java and compiled the source code using the Java Standard Edition Development Kit (Java SE JDK) 1.5.0.06. We ran it on an Intel Pentium dual core 1.8 gigahertz processor with four gigabytes of RAM.

\section{Methodologies 2 and 3: Using an Integer Programming Model}

The ILP model lies at the heart of the second solution methodology, which is aimed at finding a set of homogeneously attractive TUs within each region. The methodology begins with the generation by an algorithm of all possible TUs (i.e., clusters) formed by contiguous districts or subdistricts and subject to the condition discussed earlier-that each cluster must serve at least 15,000 and not more than 40,000 meals. An attractiveness score is then defined for each cluster (see Appendix B) using concepts similar to those developed in Appendix A. Finally, the ILP model is used to select, for each region, a set of clusters constituting a partition of the region that minimizes the difference between the most and least attractive clusters. This set is the final solution for this methodology's TU partition.

The third methodology applies the heuristic (i.e., the first methodology) using the final solution generated by the ILP model as its starting point.

\begin{tabular}{ll}
\multicolumn{2}{c}{ Initial situation } \\
\hline TU 1: $55.9 \%$ & TU 2: $44.1 \%$ \\
\hline Arica A & Iquique \\
Arica B & Pozo Almonte \\
Arica C & Huara \\
General Lagos & Pica \\
Putre & Alto Hospicio A \\
Camarones & Alto Hospicio B \\
& Camiña \\
& Colchane \\
Std. deviation & 5.9261
\end{tabular}

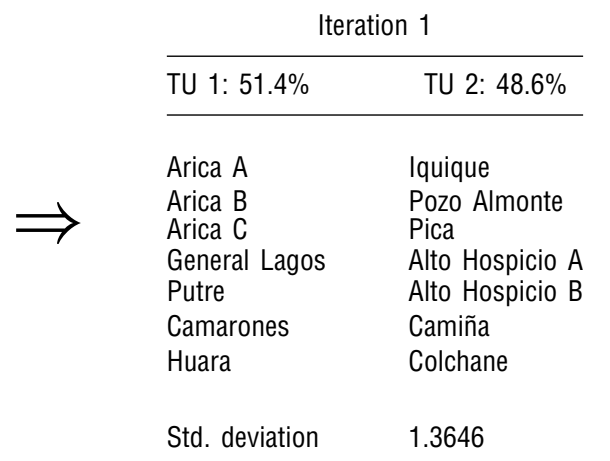

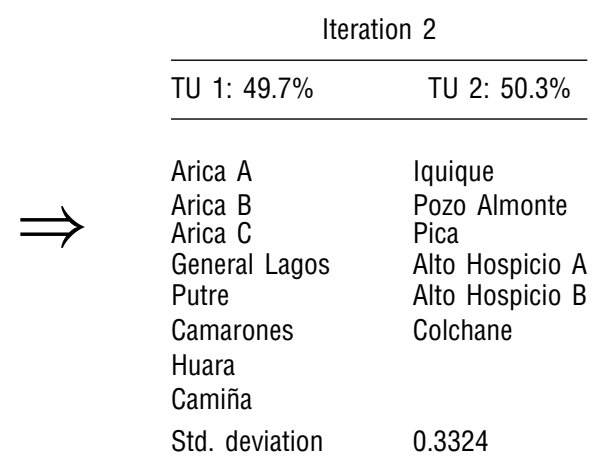

Figure 2: The tables illustrate the heuristic operation in the 1st region using a two-TU configuration. 


\begin{tabular}{|c|c|c|c|c|c|c|c|c|}
\hline \multicolumn{3}{|c|}{ Initial situation } & \multicolumn{3}{|c|}{ Iteration 1} & \multicolumn{3}{|c|}{ Iteration 2} \\
\hline TU 1: 38.05\% & TU 2: $30.37 \%$ & TU 3: $31.58 \%$ & TU 1: $35.69 \%$ & TU 2: $32.35 \%$ & TU 3: $31.96 \%$ & TU 1: $33.05 \%$ & TU 2: $32.43 \%$ & TU 3: $34.52 \%$ \\
\hline $\begin{array}{l}\text { Arica A } \\
\text { Arica B } \\
\text { General Lagos } \\
\text { Putre }\end{array}$ & $\begin{array}{l}\text { Arica C } \\
\text { Camarones } \\
\text { Huara } \\
\text { Camiña } \\
\text { Colchane } \\
\text { Alto Hospicio A }\end{array}$ & $\begin{array}{l}\text { Alto Hospicio B } \\
\text { Iquique } \\
\text { Pozo Almonte } \\
\text { Pica }\end{array}$ & $\begin{array}{l}\text { Arica A } \\
\text { Arica B } \\
\text { General Lagos } \\
\text { Putre } \\
\text { Colchane }\end{array}$ & $\begin{array}{l}\text { Arica C } \\
\text { Camarones } \\
\text { Huara } \\
\text { Camiña } \\
\text { Alto Hospicio A }\end{array}$ & $\begin{array}{l}\text { Alto Hospicio B } \\
\text { Iquique } \\
\text { Pozo Almonte } \\
\text { Pica }\end{array}$ & $\begin{array}{l}\text { Arica A } \\
\text { Arica B } \\
\text { General Lagos } \\
\text { Putre } \\
\text { Colchane } \\
\text { Pica }\end{array}$ & $\begin{array}{l}\text { Arica C } \\
\text { Camarones } \\
\text { Huara } \\
\text { Camiña } \\
\text { Alto Hospicio A }\end{array}$ & $\begin{array}{l}\text { Alto Hospicio B } \\
\text { Iquique } \\
\text { Pozo Almonte }\end{array}$ \\
\hline Std. deviation & 3.3722 & & Std. deviation & 1.6709 & & Std. deviation & 0.8785 & \\
\hline
\end{tabular}

Figure 3: The tables illustrate the heuristic operation in the 1st region using a three-TU configuration.

\section{Cluster Enumeration Algorithm}

The generation of the clusters is performed by a cluster enumeration algorithm that incorporates ideas used in solving forest harvest planning problems (Goycoolea et al. 2005, Murray and Weintraub 2002). The design of these problems involves a region that is divided into blocks, the equivalent of our clusters, that are subdivided into cells, equivalent to our districts or subdistricts. The algorithm proceeds by enumerating a priori all clusters that are feasible as regards district contiguity and number of meals. It is based particularly on the algorithm that Goycoolea et al. (2009) present, which constructs blocks of contiguous stands or zones of forested land subject to a maximum area restriction. Our approach is partially analogous to this procedure in that it uses a contiguity constraint, but replaces the area restriction with the permitted range for the number of meals.

Thus, the basic unit in our problem, a cell, is either a district or a subdistrict. As with the heuristic methodology, subdistricts are created from districts serving more than 10,000 meals by partitioning them into the smallest number of divisions, such that each serves an equal quantity no more than the mentioned figure. Also, because clusters are subject to a 15,000-meal lower bound, each will contain at least two cells. Each cell must be assigned in its entirety to a single cluster, which may include only contiguous cells. This ensures that each cluster will be connected in the graph-theory sense of connectivity-a path can always be found from one cell to any other in the same cluster without going outside the cluster.

The algorithm begins by defining all possible onecell clusters, placing them in set $S(1)$. When the algorithm starts the $(k+1)$ th iteration, the set $S(k)$ will contain all feasible clusters (satisfying the upper and lower meal-number bounds) of $k$ contiguous cells. The set $S(k+1)$ is constructed by adding every possible cell that is contiguous to any cluster in $S(k)$.

The recursion ends when no feasible clusters are left or the preset maximum number of iterations has been reached. This preset figure thus determines the maximum number of cells a cluster can contain. We found it by adding a "comfort margin" of approximately 25 percent (based on computations) to the final result generated by the heuristic method. For example, for the 1st region, because the largest TU contained eight cells, the maximum number of iterations was fixed at 10 .

The data inputs to the algorithm for each region are: the cells making up the region; the maximum number of cells that a single cluster can contain (equal to the maximum number of possible algorithm iterations); a spatial contiguity matrix (a binary matrix indicating, for any pair of cells, whether or not they are contiguous); and a meals vector for each cell that gives the number of meals for each a priori cluster.

Table 7 brings together various data relating to the algorithm. The cells and clusters formed and the scores for each cluster will be entered into the ILP model (see Appendix B).

Like the local search heuristic, we programmed the cluster enumeration algorithm in Java and compiled its source code using Java SE JDK 1.5.0.06. We ran it an Intel Pentium Dual Core 1.8 gigahertz processor with four gigabytes of RAM.

\section{Formulation of an Integer Programming Model}

We formulated an ILP model in which the clusters formed a priori by the algorithm are used as decision variables. Each cluster also has a calculated score 


\begin{tabular}{lccc} 
Region & Cells & Iterations & $\begin{array}{c}\text { Clusters formed } \\
\text { (algorithm) }\end{array}$ \\
\hline I & 14 & 10 & 3,143 \\
II & 11 & 9 & 347 \\
III & 11 & 9 & 701 \\
IV & 19 & 10 & 17,875 \\
V & 34 & 9 & 68,894 \\
VI & 31 & 9 & 607,495 \\
VII & 32 & 8 & 142,002 \\
VIII & 45 & 8 & 366,866 \\
IX & 35 & 8 & 185,706 \\
X & 34 & 8 & 62,028
\end{tabular}

Table 7: The table shows cluster enumeration algorithm data for $\mathbf{1 0}$ regions.

(see Appendix B). The objective function of the model attempts to minimize the difference between the lowest and highest scores for the TUs in any given region. This objective is a linear approximation to the nonlinearity of the standard deviation formula. The optimization model functions as a tool for comparing the goodness of the results generated by the local search heuristic, given that their respective objective functions (for minimizing the gap between best and worst TUs and for minimizing standard deviation) have similar characteristics. We also use the model in our third methodology to generate good initial solutions for the heuristic (see the Integer Programming Model + Heuristic section). We describe the model formulation below.

\section{- Variables}

$a=$ minimum score of a $\mathrm{TU}$ in a given region.

$b=$ maximum score of a TU in a given region.

$$
y_{j}= \begin{cases}1 & \text { if cluster } j \text { is selected to be a TU, } \\ 0 & \text { otherwise. }\end{cases}
$$

\section{- Parameters}

$v_{j}=$ score of cluster $j$, derived from the four criteria.

$\Omega_{i}=$ set of clusters containing cell $i$.

$P=$ average score of selected TUs in a given region.

\section{- Objective function}

$$
\min (b-a)
$$

- Constraints

$$
\begin{gathered}
\sum_{j \in \Omega_{i}} y_{j}=1 \quad \forall i \\
a \leq v_{j} * y_{j}+\left(1-y_{j}\right) * P \leq b \quad \forall j \\
y_{j} \in\{0,1\} \quad \forall j \\
a, b \geq 0 .
\end{gathered}
$$

The aim of the objective function (1) is to obtain relatively homogeneous TUs within each region by minimizing the gap between their highest (b) and lowest (a) scores.

As for the constraints, Equations (2) state that of all the clusters containing a given cell, exactly one must be chosen as a TU. Inequalities (3) set the permitted range of the TU scores. The parameter $P$ is the average score of the selected TUs; we include it here to maintain the logical validity of Equation (3) in the case in which cluster $j$ is not selected. Because neither this average nor the number of TUs in a region is known a priori, different values of $P$ are initially used for each region and the final value eventually chosen will be the optimal one giving the best objective function. Note also that the value of $P$ can be estimated if the number of TUs is known, given that the TU values in any partition add up to a figure close to 100 (see Appendix B). For example, if we estimate that a region will have between two and five TUs, the $P$ values used will be 20 (100/5), 25 (100/4), 33 (100/3), and $50(100 / 2)$.

Finally, Constraint (4) ensures that $y_{j}$ is a binary variable, whereas Constraint (5) requires that $a$ and $b$ are positive real numbers.

We programmed the ILP model in GAMS 22.1 and solved it to optimality by CPLEX 10.0. We used the same hardware setup that we used for running the heuristic and the cluster enumeration algorithm.

\section{Integer Programming Model + Heuristic}

In our third methodology, the solution generated by the ILP model, which minimizes the gap between best and worst TUs, is used as the initial solution for the local search heuristic, which minimizes their standard deviation. The TU scores are calculated using the procedure described in Appendix A.

The reasoning behind this approach is that the ILP model and the heuristic complement each other well 


\begin{tabular}{|c|c|c|c|c|c|c|c|}
\hline \multirow[b]{2}{*}{ Region } & \multicolumn{4}{|c|}{ Standard deviation } & \multicolumn{3}{|c|}{ Percentage improvement (\%) } \\
\hline & Original & Heuristic & Model & $\mathrm{M}+\mathrm{H}$ & $\mathrm{O} \mathrm{v} / \mathrm{s} \mathrm{H}$ & $0 \mathrm{v} / \mathrm{s} \mathrm{M}$ & $\mathrm{O} v / \mathrm{s} \mathrm{M}+\mathrm{H}$ \\
\hline I & 5.93 & 0.33 & 0.29 & 0.29 & 94.40 & 95.11 & 95.11 \\
\hline II & 0.18 & 0.18 & 0.18 & 0.18 & 0.00 & 0.00 & 0.00 \\
\hline III & 0.74 & 0.19 & 0.19 & 0.19 & 74.57 & 74.57 & 74.57 \\
\hline IV & 5.94 & 1.81 & 0.88 & 0.88 & 69.49 & 85.26 & 85.26 \\
\hline V & 1.47 & 0.68 & 0.99 & 0.93 & 53.45 & 32.79 & 36.85 \\
\hline $\mathrm{VI}$ & 3.27 & 0.47 & 0.16 & 0.16 & 85.75 & 95.19 & 95.19 \\
\hline VII & 4.05 & 0.41 & 0.53 & 0.27 & 89.88 & 86.81 & 93.33 \\
\hline VIII & 2.44 & 0.60 & 0.87 & 0.53 & 75.48 & 64.24 & 78.31 \\
\hline IX & 3.90 & 0.40 & 0.03 & 0.03 & 89.85 & 99.14 & 99.14 \\
\hline$x$ & 1.04 & 0.84 & 0.88 & 0.88 & 19.21 & 15.28 & 15.28 \\
\hline
\end{tabular}

Table 8: The table shows a comparison of standard deviations for TUs generated by the three methodologies and the pre-2007 configuration. It also shows the percentage improvement over the original configuration, obtained by dividing the modulus of the difference between the two standard deviations being compared by the highest standard deviation. In each case, the TU attractiveness levels were calculated for each final solution using Methodology 1 (see Appendix A).

when the objectives to be satisfied by the solution are similar. Because the initial solution provided by the model is optimal for minimizing the difference between the best and worst TU scores, the heuristic should not significantly change it for regions in which we can improve the standard deviation of the TUs.

\section{Results}

In this section, we present the results obtained from applying the three methodologies described above. For some regions, the distribution of districts generated by any of the three diverges greatly from the pre2007 distribution because of the highly heterogeneous nature of their TUs, whereas for other regions the difference is very small because the TUs were already relatively homogeneous.

Table 8 analyzes the standard deviations and uses the following headings:

- Original $(\mathrm{O})$ : the standard deviation of the pre2007 TU configuration.

- Heuristic $(\mathrm{H})$ : the standard deviation of the configuration generated by the heuristic, whose objective is to minimize the standard deviation between the TUs within a single region.

- Model (M): the standard deviation of the configuration generated by the ILP model, whose objective is to minimize the difference between the highest and lowest TU scores within a single region.
- $\mathrm{M}+\mathrm{H}$ (model + heuristic): the standard deviation of the configuration generated by using the ILP model result as the initial solution of the heuristic, whose objective is to minimize the standard deviation.

In all regions except the second (whose original configuration was already homogeneous), our proposed methodologies generate better solutions, satisfying the main objective of the study, which was to identify a homogeneously attractive set of TUs in each region. Table 9 compares the differences between best and worst TU scores and uses the following headings:

- Original $(\mathrm{O})$ : differences between best and worst TU scores of the .pre-2007 TU configuration.

- Heuristic (H): differences between best and worst TU scores of the configuration generated by the heuristic, whose objective is to minimize the standard deviation between the TUs within a single region.

- Model (M): differences between best and worst TU scores of the configuration generated by the ILP model, whose objective is to minimize the difference between the highest and lowest TU scores within a single region.

- $\mathrm{M}+\mathrm{H}$ (model + heuristic): differences between best and worst TU scores of the configuration generated by using the ILP model result as the initial solution of the heuristic, whose objective is to minimize the standard deviation.

Recall that we could not use the Appendix A methodology to calculate the attractiveness of an isolated 


\begin{tabular}{|c|c|c|c|c|c|c|c|}
\hline \multirow[b]{2}{*}{ Region } & \multicolumn{4}{|c|}{ Differences (max-min) } & \multicolumn{3}{|c|}{ Percentage improvement (\%) } \\
\hline & Original & Heuristic & $M+H$ & Model & $\mathrm{O} \mathrm{v} / \mathrm{s} \mathrm{H}$ & $\mathrm{O} \mathrm{v} / \mathrm{s} \mathrm{M}+\mathrm{H}$ & $0 \mathrm{v} / \mathrm{s} \mathrm{M}$ \\
\hline I & 12.70 & 0.55 & 0.04 & 0.04 & 95.67 & 99.69 & 99.69 \\
\hline$\|$ & 0.20 & 0.20 & 0.20 & 0.20 & 0.00 & 0.00 & 0.00 \\
\hline III & 2.55 & 1.02 & 1.02 & 1.02 & 60.14 & 60.14 & 60.14 \\
\hline IV & 46.49 & 3.36 & 1.58 & 1.58 & 92.77 & 96.60 & 96.60 \\
\hline $\mathrm{V}$ & 12.62 & 4.82 & 1.47 & 1.26 & 61.83 & 88.39 & 89.99 \\
\hline $\mathrm{VI}$ & 40.84 & 2.58 & 0.37 & 0.37 & 93.68 & 99.10 & 99.10 \\
\hline VII & 63.48 & 1.14 & 1.53 & 0.17 & 98.20 & 97.59 & 99.73 \\
\hline VIII & 72.86 & 2.87 & 2.49 & 2.49 & 96.06 & 96.59 & 96.59 \\
\hline IX & 47.09 & 1.37 & 0.13 & 0.13 & 97.10 & 99.72 & 99.72 \\
\hline$x$ & 3.81 & 2.51 & 2.25 & 2.25 & 34.04 & 40.89 & 40.89 \\
\hline
\end{tabular}

Table 9: The table shows a comparison of the differences between best and worst TU scores for results generated by the three methodologies and the pre-2007 configuration. The percentage improvement is derived by dividing the modulus of the difference between the two gaps being compared by the highest gap. We calculated the TU attractiveness levels for each final solution using the second methodology (see Appendix B).

cluster, given that it calculates the attractiveness of a TU with a known global configuration of the region; in the ILP model, we calculated a cluster's attractiveness without knowing the rest of the region's configuration. The best solutions are clearly those obtained using the integer programming model. Again, in every region except the second, the three proposed methodologies generate solutions that are superior to the pre-2007 configuration.

Also worth noting is that under any analysis, the three methodologies yield more homogeneous scores for the TUs in every region than those for the original configuration (except the 2nd region) and thus demonstrate the robustness of our models.

The number of TUs obtained for the majority of regions was the same in all the methodologies (see Table 10). This minimal variation was to be expected given that an estimate of this factor is easily obtained by calculating the average number of meals per TU. Knowing the TU meal numbers' upper and lower bounds helps in making this calculation. Note also that the total number of TUs for the country generated by our methodologies dropped by 25 units- 18 percent compared to the 136 TUs in the pre-2007 configuration, including the three regions excluded from this study.

The lower number of TUs was an additional benefit of our study. In JUNAEB's view, a slight reduction was desirable for administrative reasons because it facilitates management control and improves the functioning of the tender process.
The solution times of the proposed methodologies varied considerably. The heuristic's run times for each initial solution were a few milliseconds for regions with a small number of districts, increasing to a few seconds for the larger ones. This performance is influenced by the configuration of the initial solution, the number of districts in the region, and their distribution within it. The impact of districts serving many meals differs greatly, depending on whether they are bunched together or widely dispersed.

The cluster enumeration algorithm also generated its results rapidly. For most regions, the run times for creating the feasible clusters were under one minute. They reached 20 minutes for only three regions; in

Region TUs (original configuration) TUs (heuristic) TUs (ILP model)

$\begin{array}{lrrr}\text { I } & 2 & 2 & 2 \\ \text { II } & 2 & 2 & 2 \\ \text { III } & 2 & 2 & 2 \\ \text { IV } & 5 & 3 & 3 \\ \text { V } & 10 & 8 & 7 \\ \text { VI } & 9 & 5 & 5 \\ \text { VII } & 10 & 5 & 6 \\ \text { VIII } & 17 & 10 & 10 \\ \text { IX } & 9 & 7 & 7 \\ \text { X } & 11 & 8 & 8 \\ \text { Total } & 77 & 52 & 52\end{array}$

Table 10: The table shows the number of TUs in the pre-2007 configuration and under our methodologies. Note that $\mathrm{M}+\mathrm{H}$ is not shown because the number of TUs will always equal that of the ILP model. 
one case, they reached two hours. The run times for the integer programming model (for each fixed $P$, the average TU attractiveness score) did not exceed one hour, with the exception of the 8th region, in which, for certain $P$ values, they extended to five hours because of the number of cells and the many possible combinations. For the 1st, 2nd, and 3rd regions, solutions were generated in no more than 60 seconds.

Finally, when the ILP model result is used as the initial solution of the heuristic, run times are similar to those for the model because the heuristic yields its results in seconds.

\section{Conclusions and Practical Impact}

This paper applied different OR methodologies to determining a new configuration of the geographical divisions (TUs). The goal was to create a set of such units in each region of the country that would be equally attractive to bidding suppliers, thereby lowering the bankruptcy risk and uncertainty they faced. This homogenization of the TUs would also guarantee that schools in each region received similar quality meals. The combinatorial nature of the tender process makes an appropriate definition of the units essential.

The TU configurations that the three proposed methodologies generated for any given region were, for the majority of regions, highly similar. The heuristicbased approach in particular yielded satisfactory results very rapidly. The pre-2007configuration caused major problems in the regions in which very attractive districts were awarded by the tender process as single TUs. Bidders focused their interest on those districts at the expense of the other districts in these regions, whereas less attractive units were assigned to suppliers who later ran into serious problems in providing the contracted services.

The configurations our methodologies produced distributed attractive districts among more than one TU so that various units would have a significant number of meals on which the firms could bid. By eliminating unattractive TUs, supplier bankruptcy risk declined appreciably. This is the main contribution of our study. Lizette Vega, an official in charge of school meal programs at JUNAEB, has confirmed its success. "Five companies that were awarded mealsupply contracts went broke between 2004 and 2007," she noted, adding that "the new configuration should help ensure this does not happen again. In fact, since 2007 no bankruptcies have occurred" (L. Vega, pers. comm.).

These bankruptcies had endangered the normal functioning of the food industry and posed a serious threat to the contracting model that JUNAEB used. Restoring meal service in each case resulted in additional annual costs to the government of approximately USD 1 million over the original winning bid price (see Table 2) and enormous social costs because of the temporary interruptions in meal service.

The agency also pointed out that the main objective of the TU configuration solution has been fully met, given that the new configuration generated by the heuristic methodology has triggered greater interest among potential suppliers in bidding on the new TUs, and the bids submitted have been more competitive and less risky.

Although improving the attractiveness of one TU necessarily implies worsening the attractiveness of another, we expect an overall increase in the efficiency of both JUNAEB and the suppliers. The key objective is to ensure that no TU is unattractive because this significantly increases the risk of bankruptcy, thus jeopardizing the entire auction.

The creation of equally attractive TUs is also advantageous to the suppliers because it simplifies calculating the cost of the different bids they submit. With homogeneous TUs, a calculation can be made for any single TU and then extended almost directly to all other TUs in the same region, in contrast to the previous situation in which costs among TUs varied widely. Furthermore, homogenized TUs mean that bidders no longer have to submit offers on units whose conditions are difficult to evaluate, a situation that added considerably to supplier risks.

Amalia Cornejo, head of JUNAEB's Social Program Department, remarked,

In our view, the homogenization of the TUs has helped reduce the overall cost to the Chilean government of the tender process. It's true that the quantitative impact of the reconfiguration is difficult to measure in terms of countrywide unit meal costs because at the same time this change was implemented JUNAEB significantly increased its budget and improved meal quality, which caused this cost factor to rise. What we can 
report, however, is that 25 percent more suppliers participated in the 2008 tender compared to 2005, when contracts for the same regions were tendered, and both the quantity and quality of the bids have gone up. (A. Cornejo, pers. comm.)

The key factors in the creation of the new TU configurations that would satisfy the objectives of both JUNAEB and the supplier firms were the definition of the principal criteria that make a TU attractive and low risk, the quantification of these criteria, and the use of OR techniques to produce configurations with homogeneous attractiveness levels. A manual approach to defining TU configurations, such as JUNAEB used before 2007, could not achieve the same results.

\section{Electronic Companion}

An electronic companion to this paper, which includes Appendices A and $\mathrm{B}$, is available as part of the online version that can be found at http://interfaces.pubs .informs.org/ecompanion.html.

\section{Acknowledgments}

We wish to thank the Millennium Institute for Complex Engineering Systems for its support in carrying out this study; the officials at JUNAEB, especially Amalia Cornejo and Lizette Vega, for their valuable collaboration; Andrés Weintraub for his collaboration in bringing this project to fruition; Jaime Catalán and Daniel Yung for their valuable support in justifying and measuring the impact of our proposals; Flavia Bonomo, Mario Guajardo, Pablo Rey, and Kenneth Rivkin for suggestions that improved the quality of the final product; Nicolás Maluenda and Ronald Poillot for their assistance in implementing the local search heuristic; and both anonymous referees for their valuable suggestions. The first author was partially financed by FONDECYT project number 1080286 (Chile) and by ANPCyT PICT-200700518 and UBACyT grant X069 (Argentina).

\section{References}

Caro, F., T. Shirabe, M. Guignard, A. Weintraub. 2004. School redistricting: Embedding GIS tools with integer programming. J. Oper. Res. Soc. 55(8) 836-849.

Catalan, J., R. Epstein, M. Guajardo, D. Yung, C. Martínez. 2009. Solving multiple scenarios in a combinatorial auction. Comput. Oper. Res. 36(10) 2752-2758.

Cramton, P., Y. Shoham, R. Steinberg, eds. 2006. Combinatorial Auctions. MIT Press, Cambridge, MA.

Decarolis, F. 2009. When the highest bidder loses the auction: Theory and evidence from public procurement. Working paper, Bank of Italy, Economic Research Department, Rome.
Epstein, R., L. Henríquez, J. Catalán, G. Weintraub, C. Martínez. 2002. A combinatorial auction improves school meals in Chile. Interfaces 32(6) 1-14.

Epstein, R., L. Henríquez, J. Catalán, G. Weintraub, C. Martínez, F. Espejo. 2004. A combinatorial auction improves school meals in Chile: A case of OR in developing countries. Internat. Trans. Oper. Res. 11 593-612.

Goycoolea, M., A. Murray, J. Vielma, A. Weintraub. 2009. Evaluating approaches for solving the area restriction model in harvest scheduling. Forest Sci. 55(2) 149-165.

Goycoolea, M., A. Murray, F. Barahona, R. Epstein, A. Weintraub. 2005. Harvest scheduling subject to maximum area restrictions: Exploring exact approaches. Oper. Res. 53(3) 490-500.

IBM. 2009. Latin America school lunch program auction system for school food services. Accessed June 1, 2010, http:// public.dhe.ibm.com/common/ssi/pm/ab/n/wsc14149usen/ WSC14149USEN.PDF.

Milgrom, P. 2007. Putting Auction Theory to Work. Cambridge University Press, Cambridge, UK.

Murray, A., A. Weintraub. 2002. Scale and unit specification influences in harvest scheduling with maximum area restriction. Forest Sci. 48(4) 779-789.

Olivares, M., G. Weintraub, D. Yung, R. Epstein. 2010. The design of combinatorial auctions for procurement: An empirical study of the Chilean school meals auction. Accessed October 5, 2010, http://www.columbia.edu/ gyw2105/GYW/Main_Page_files / paperCA_100429-post-wFigures.pdf.

Parlane, S. 2003. Procurement contracts under limited liability. Econom. Soc. Rev. 34(1) 1-21.

Saaty, T. 1980. The Analytic Hierarchy Process. McGraw-Hill, New York.

Taylor, R., M. Vasu, J. Causby. 1999. Integrated planning for school and community: The case of Johnston County, North Carolina. Interfaces 29(1) 67-89.

Thaler, R. 1988. The winner's curse. J. Econom. Perspect. 2(1) 191-202.

Amalia Cornejo, Head of the Social Programs Department, JUNAEB-CHILE, writes: "The purpose of this letter is to describe the impact of the Operations Research techniques developed by the researchers of the Universidad de Chile's Operations Management Center (CGO) in order to determine a new configuration of the country in territorial units to be used in auctions for the school meal program of our organization.

"Junta Nacional de Auxilio Escolar y Becas (JUNAEB) is the Chilean Government entity whose purpose is to help socially vulnerable children and teenagers to enter and remain in the school system. To this effect, this entity has programs in many areas, such as meals, school health, mental health, dental health, housing, recreation, school materials and grants intended to provide comprehensive assistance 
making effective equality of opportunity at educational level. These actions benefit around two million students throughout the country.

"JUNAEB's school meal program consists of providing different kinds of meals for school children. JUNAEB holds annual auctions to choose the catering companies which are going to provide the service. For this purpose, the country is divided into Territorial Units (TU). These are the items of the auction, meaning that a company which awards a TU will provide the catering service in all the schools contained in that TU. In practice, companies can present multiple combinatorial bids, including more than one TU, thus this process is a combinatorial auction. The Chilean Government invests about 500 million dollars a year in this program.

"The main problem with the territorial configuration that was used until 2007, affecting both the companies and the JUNAEB, was the disparity of incentives among the TU, since only the use of a geographical criterion and some equity as per the number of meals in each one of the territories were taken into account.

"This is why we decided to design a new territorial structure to obtain an homogeneous configuration, with relatively similar units. That is, to decrease the gap between attractive territories and those not so attractive to the companies. This way, each school within a single region may receive proper meals of a similar quality.

"To address this problem, we consulted specialists in Operations Research of Universidad de Chile (who have worked with us since 1997 developing a mathematical model which helps us to award the auctions year after year), to design a model which could allow us to obtain a new territorial configuration. As result of their research, they proposed this new configuration to us in 2007 that meets our goals and companies' requirements. They used Operations Research methods to consider the different incentives the companies have to bid on a TU, and carried out a new configuration, much more homogeneous than the one we used before. Clearly, if we had approached this problem manually, it would not have been possible to obtain such an excellent result.

"Since 2007, we have used the new configuration of TU proposed by the academic team of Universidad de Chile. In addition, since the market is dynamic, we have asked them to repeat this analysis on a periodical basis in order to update the territorial configuration when significant changes are reported." 